\title{
Contribution de la biodiversité à l'alimentation
}

\section{Serge Bahuchet, Françoise Aubaile et Ricardo Ávila Palafox}

\section{OpenEdition}

\section{Journals}

Édition électronique

URL : http://journals.openedition.org/ethnoecologie/1138

DOI : $10.4000 /$ ethnoecologie. 1138

ISSN : 2267-2419

\section{Éditeur}

Laboratoire Eco-anthropologie et Ethnobiologie

\section{Référence électronique}

Serge Bahuchet, Françoise Aubaile et Ricardo Ávila Palafox, « Contribution de la biodiversité à l'alimentation », Revue d'ethnoécologie [En ligne], 2 | 2012, mis en ligne le 12 novembre 2013, consulté le 22 septembre 2020. URL : http://journals.openedition.org/ethnoecologie/1138; DOI : https:// doi.org/10.4000/ethnoecologie.1138

Ce document a été généré automatiquement le 22 septembre 2020.

\section{c) (i)}

Revue d'ethnoécologie est mis à disposition selon les termes de la licence Creative Commons Attribution - Pas d'Utilisation Commerciale - Pas de Modification 4.0 International. 


\title{
Contribution de la biodiversité à l'alimentation
}

\author{
Serge Bahuchet, Françoise Aubaile et Ricardo Ávila Palafox
}

1 En décembre 2008, une quarantaine de chercheurs et de doctorants de plusieurs pays du monde se sont réunis à Paris au Musée de l'Homme, sous l'égide du CNRS, $\mathrm{du}$ Muséum national d'Histoire naturelle et de l'ICAF, pour échanger les résultats de leurs recherches et de leur expérience sur le thème de la contribution de la biodiversité à l'alimentation humaine. Ce colloque a constitué le $26^{\mathrm{e}}$ symposium de l'International Commission for the Anthropology of Food.

2 Nous avons souhaité offrir la publication de ce colloque à Igor de Garine, au moment où il quitte la présidence de l'ICAF, en reconnaissance de son rôle infatigable pour promouvoir l'anthropologie de l'alimentation dans la communauté scientifique.

3 Les anthropologues de l'alimentation réunis au Musée de l'Homme sont d'abord des ethnologues qui travaillent à l'échelle du village, du foyer, de la personne. Cela les distingue des sociologues, qui œuvrent à l'échelle de la population, ou des économistes, dont l'approche embrasse la région, le pays, voire le monde. Le regard qu'ils portent sur le phénomène alimentaire est ainsi singulier et pénétré de détails. 

complémentaires en se basant sur diverses aires culturelles : la domestication de la biodiversité végétale (Nouvelle-Guinée), des régimes alimentaires complexes où la diversité biologique est prépondérante (Laos, Congo), des exemples de transformations historiques (France, Asie centrale), des aliments issus de la cueillette (les champignons en France), l'échelle du paysage considéré comme « nourricier » (Grande Bretagne), les tentatives de conservation de la biodiversité par la création d'une réserve de la biosphère face à l'expansion de l'agriculture (Mexique), enfin des mécanismes de protection du patrimoine, à travers un produit et ses paysages (la Tequila au Mexique), ou bien l'alimentation dans son intégralité et prise comme modèle (la Méditerranée).

7 Les résultats présentés dans ces communications s'insèrent dans un contexte international où l'opinion sur l'alimentation évolue fortement. En premier lieu, oui, la biodiversité, tant sauvage que domestique, est présente et importante dans l'alimentation humaine, comme cela a déjà été largement démontré dans de nombreuses publications, par exemple dans des ouvrages collectifs sur l'Afrique centrale (Froment et al. 1996) ou sur les forêts tropicales (Hladik et al. 1993). On citera les plantes de cueillette, dont l'importance dans le monde contemporain a été bien documentée, par exemple au Cameroun (Garine 1996) et même en Europe (en Turquie, Dogan et al. 2004, en Espagne, Tardío et al. 2005, en Italie, Ranfa et al. 2013...).

8

Il a certes été souligné que la biodiversité est nécessaire à une alimentation équilibrée, mais il a aussi été montré que d'autres facteurs entrent en jeu - économiques, sociaux...- qui conditionnent tout autant cet équilibre nutritionnel. En même temps, on observe partout de très nettes transformations qui vont toutes vers une déperdition de la diversité alimentaire avec de forts risques d'appauvrissement nutritionnel. Partout dans le monde, le rôle de la biodiversité décroît. Ainsi, lorsque l'on connaît l'importance très ancienne des plantes rudérales de cueillette dans l'alimentation mexicaine, les quelites (Castro Lara et al. 2011 recensent 205 espèces sur l'ensemble du pays ; Picó \& Nuez 2000), on est frappé par leur absence des plats consommés dans des régions au fort développement agricole comme le Jalisco, dans l'Occident mexicain, et ceci à cause de l'usage généralisé des désherbants dans les parcelles cultivées. D'autres auteurs ont pu analyser comment d'anciens chasseurs-cueilleurs d'Australie passent d'un régime alimentaire entièrement basé sur l'usage de la biodiversité spontanée à un régime de type occidental, s'approvisionnant dans le commerce (Smith \& Smith 1999).

Dans ce processus de transformation, nous avons relevé plusieurs questions différentes :

10 La part de la nourriture auto-produite dans la diète, par rapport à la nourriture achetée, ne cesse de décroître, en relation avec la monétarisation désormais quasiuniverselle des modes de vie; entre alors en jeu l'influence d'aliments exogènes considérés comme prestigieux.

11 À l'échelle mondiale, l'urbanisation croît considérablement. Ce phénomène urbain a deux aspects: d'une part le problème de la nourriture des citadins, d'autre part 
l'influence de l'économie urbaine sur les zones rurales. Dans le premier cas, la question se pose de la distribution et de la diffusion des produits alimentaires, diversifiés ou non (par exemple dans nos régions européennes à travers la concentration dans les supermarchés). Dans le second, l'approvisionnement des villes pèse sur le système économique des régions rurales tout en modifiant les modes de vie paysans, notamment leurs styles alimentaires. L'approvisionnement croissant des pays du sud par les pays du nord, notamment par l'écoulement de leurs surplus de production et par l'imposition de règles de libre échange contraignantes et défavorables, aggrave ces transformations.

12 Les mêmes règles commerciales rendent certains produits alimentaires, parmi les plus valorisés dans les systèmes traditionnels (viandes, féculents, huiles ou graisses...), beaucoup moins coûteux à acheter qu'à produire, déséquilibrant ainsi les systèmes de production locaux.

13 La monétarisation et la commercialisation croissantes induisent des changements économiques, liés ou non à l'influence des villes, notamment par la part des cultures de rente qui empiètent sur les "meilleures" terres et celles consacrées aux cultures vivrières. Cela s'effectue au détriment de la biodiversité et entraîne de graves problèmes de sécurité alimentaire dans les régions rurales. Une agriculture durable semble encore hors de portée dans de nombreuses régions du monde...

Les transformations économiques vont de pair avec une rupture dans la transmission vers les jeunes générations des pratiques alimentaires et des goûts. Comment et par qui se transmettent les préparations alimentaires? La question de l'éducation est centrale et la scolarisation, nécessaire et utile sur de très nombreux domaines, est périlleuse en ce qui concerne la connaissance et l'usage de la biodiversité, comme les études ethnobiologiques l'ont déjà malheureusement amplement montré.

On s'est aussi interrogé sur l'existence de politiques publiques favorables à la diversité alimentaire, à travers la biodiversité. Deux tendances coexistent, la protection des espaces dans diverses aires protégées (parcs régionaux, réserves de biosphères, etc.), ou bien la protection des produits eux-mêmes à travers les législations d'indications géographiques (cf. Bérard \& Marchenay 2004). Si ces deux tendances sont porteuses d'espoir, le moins que l'on puisse dire, c'est qu'elles offrent des possibilités mais que les résultats actuels ne dessinent pas encore une image claire, ni pour la protection de la biodiversité, ni pour la diversité alimentaire, tant les facteurs sociopolitiques en jeu sont nombreux et les facteurs économiques prégnants.

Cela rejoint néanmoins la question déjà évoquée de l'agriculture durable : comment maintenir une diversité biologique élevée, celle que l'on nomme désormais "biodiversité ordinaire », c'est-à-dire hors des aires protégées, avec une vie rurale équilibrée, seule condition pour construire une gestion durable de la biodiversité ?

Comment aller plus loin?

18 Le contexte, avouons-le, est particulièrement violent, alors que le monde est soumis à cinq crises simultanées : crise de la biodiversité, crise énergétique, crise alimentaire, crise financière et changement climatique... Tout cela pose à nouveau, très vivement et douloureusement, le problème de la pauvreté et celui de la sécurité alimentaire, désormais dans toutes les régions du globe.

Toutefois, face à ce tableau effrayant, quelques lueurs d'espoir existent, car on assiste à une prise de conscience qui conduit à une forme de reconnaissance officielle, à la 
faveur de l'entrée en vigueur de la Convention de la Diversité Biologique (1992). Il est éclairant de voir comment l'agriculture et l'alimentation se sont progressivement insérées dans la diversité biologique. Pendant les premières années, furent essentiellement prises en compte les "espèces domestiquées ou cultivées " et leur diversité génétique, ainsi que les impacts négatifs de l'agriculture productiviste sur les espèces et les écosystèmes (texte de la Convention de $1992^{1}, 2^{\mathrm{e}}$ Conférence des Parties en 1995). En 1996, la $3^{\text {e }}$ Conférence des Parties ${ }^{2}$ encourage les États signataires (les "Parties») à développer des programmes nationaux sur la diversité biologique agricole, dans le cadre de l'article $6 \mathrm{~b}$ de la Convention qui concerne sans précision « les programmes et politiques sectoriels». En effet, aucun article de la Convention ne concerne spécifiquement la biodiversité agricole. Il faut attendre la $5^{\mathrm{e}} \mathrm{CdP}^{3}$, en 2000 , pour qu'un programme de travail pluriannuel sur la diversité biologique agricole soit défini en détail. En 2006, la $8^{\mathrm{e}} \mathrm{CdP}^{4}$ prend une décision qui change fortement l'orientation : l'insistance se déplace de la biodiversité agricole vers l'importance de la biodiversité pour l'alimentation et la nutrition. Il est affirmé par exemple: “Considering the difficulty in precisely indentifying optimal diets, a diversity of food from plants and animals remains the preferred choice for human health." Les documents préparatoires à la décision font explicitement référence au modèle proposé par Johns \& Sthapit (2004), dans lequel la conservation de la biodiversité, les traditions socioculturelles, le statut nutritionnel et sanitaire et la création de revenus sont intrinsèquement liés. Il est alors fait appel à la FAO, l'organisation des Nations Unies pour l'alimentation et l'agriculture, pour appuyer la mise en œuvre de ces décisions. Celle-ci, par exemple, y répond en rédigeant en 2011 un gros rapport Biodiversity for food and agriculture (Galluzzi et al. 2011).

Le regard s'est aussi déplacé en ce qui concerne les régimes alimentaires, et les recommandations ne portent plus sur l'apport énergétique global - désormais assuré partout par les aliments "modernes » et d'origine commerciale - mais sur l'équilibre nutritionnel. Et c'est dans ce contexte que l'importance de la biodiversité pour l'apport en micro-nutriments est primordiale. Comme l'expriment Johns \& Eyzaguirre (2006 : 182) :

Globalization of food production and supply poses unprecedented challenges to the health and well-being of populations of developing countries. While levels of hunger have decreased and per capita energy consumption has risen consistently in most countries, inexpensive foodstuffs produced by large-scale agriculture can have adverse affects on diet quality, undermine the self-sufficiency of small-scale farmers and threaten environmental sustainability.

Si la faim (manque d'énergie) a diminué, sauf dans les régions en guerre, la malnutrition, c'est-à-dire des régimes alimentaires déséquilibrés, ne baisse pas. On parle désormais de hidden hunger, la faim insoupçonnée (Kennedy et al. 2003), tant les carences en micronutriments sont présentes pour des centaines de millions de personnes à travers le monde. C'est alors que les produits de la biodiversité prennent toute leur importance. Ainsi les végétaux sauvages apporteront des compléments alimentaires nécessaires (Flyman \& Afolayan 2006) ; les produits de la petite chasse prémunissent les enfants malgaches des anémies (Golden et al. 2011) tout comme la consommation du gibier apportent des vitamines A, D et $\mathrm{E}$ aux adultes Inuit (Kuhnlein et al. 2006). Plus encore, pour les plantes cultivées, la combinaison de plusieurs cultivars assure un apport diversifié d'acides aminés complémentaires, du fait de la diversité des compositions alimentaires au niveau variétal (Kennedy \& Burlingame 2003 pour le riz ; Burlingame et al. 2009). 
us globalement, non seulement l'alimentation équilibrée est tributaire de la biodiversité, mais encore il importe de modifier l'agriculture et d'en favoriser la diversification: " agricultural biodiversity is essential for a sustainable improvement in food and nutrition security", proclament Frison et al. (2011). On peut dès lors apprécier à sa juste valeur le développement de l'agroécologie, telle qu'elle est explicitée par l'heureuse et prometteuse expression «l'écologie des systèmes alimentaires ", « ecology of food systems » (Francis et al. 2003).

Une telle démarche impose que les communautés se soustraient aux importations d'aliments au profit de productions locales, renforçant ainsi leur autonomie alimentaire (Bellows \& Hamm 2001). Ces activités n'excluent nullement artisanat et commerce, c'est-à-dire production, transformation et commercialisation des produits alimentaires. C'est la logique qui sous-tend la définition des systèmes agroalimentaires localisés (Syal) basés sur les relations entre aliments et territoire, un concept intégrateur qui prend en compte l'organisation et le fonctionnement d'un groupe d'activités productives, sociales et culturelles (Muchnik 2012).

En dehors du cadre académique et officiel, au niveau de la société civile, des forces de réaction se manifestent. On pensera ainsi au mouvement Slow Food, lancé en Italie en 1989 et qui est implanté désormais dans 150 pays ${ }^{5}$ pour promouvoir une alimentation saine et goûteuse avec le développement des cultures locales (Petrini 2005). Tout récemment, Slow Food et la FAO ont joint leurs efforts. En signant cet accord, le Directeur général de la FAO, M. José Graziano da Silva, a expliqué :

Slow Food et la FAO partagent une même vision d'un monde durable d'où la faim serait exclue et où la biodiversité serait sauvegardée pour les générations futures. L'accord signé aujourd'hui, en ouvrant la voie à de nombreuses initiatives conjointes majeures, nous rapproche un peu plus de cet objectif. (15 mai 20136).

À une autre échelle, on assiste à une certaine transformation de l'alimentation urbaine, tant dans les villes surpeuplées et désorganisées des pays en développement, que dans certains quartiers des villes des pays à forts revenus. Dans les villes africaines, telles que Tananarive, Dakar, Abidjan, où l'émigration est très forte, c'est un moyen de combattre la vie chère pour des populations mal insérées et vite démunies (Ahahan 2010). Les jardins potagers, partagés ou collectifs, se répandent aujourd'hui dans de nombreuses villes de la planète du sud, en Afrique, en Asie, comme du nord aux États Unis (Baudry 2011), au Canada, au Mexique, en Europe, à tel point que l'on peut presque parler d'une naissante «agriculture urbaine». Ainsi à New York, l'immense Brooklyn Grange, installée sur deux toits, produit 18 tonnes de légumes et de fruits par an (dépêche AFP, 17/05/2013 à 11h15). En Angleterre, à partir des initiatives de la commune de Todmorden (quasi-autosuffisante en fruits et légumes à partir de potagers plantés dans les lieux publics) se répand le programme Incredible edible ${ }^{7}$.

Dans ce monde en mouvement, quel peut être l'apport des anthropologues de l'alimentation ? Face aux changements et aux politiques de développement, il faut bien reconnaître que les recommandations émises par Igor de Garine (1972:162), il y a déjà 40 ans, restent d'actualité :

Whatever may be the philosophy underlying attempts towards economic and social development, only two main positions can be held in the field of food and nutrition. Either an attempt should be made towards promoting nutritional well-being, taking care to avoid destroying the cultural values of a society, which may actually result in slowing-down and decreasing the efficiency of a program. Or, since our own industrial society has reached the highest technological efficiency in human 
history, we wish to apply it readily to developing countries and obtain fast, concrete results, even if cultural equilibriums and values are destroyed in the process. In both cases, efficiency implies a refined analysis of the socio-cultural aspects of nutritional behavior. On pourra rappeler ici le but que se
l'anthropologie de l'alimentation (ICAF) :

The aims of the Commission are:

- To assist world anthropologists in their basic and applied research on food.

- To encourage indigenous expertise on food problems in developing countries.

- To promote the collaboration of anthropologists with institutions and individual experts from related fields of study.

- To stimulate education and training in the anthropology of food. ${ }^{8}$

Les articles proposés ici suggèrent quelques réflexions. Le premier apport serait celui de dresser un état des lieux, permettant de «capitaliser » les connaissances acquises jusqu'à présent. On peut rappeler les grandes étapes, depuis les études pionnières du $20^{\mathrm{e}}$ siècle qui ont mis en évidence les principes, la logique et l'équilibre des régimes alimentaires paysans, «traditionnels» (parmi d'autres Richards 1932, Rodahl 1964, Oomen 1971, Norgan et al. 1974); elles furent suivies par des études quantitatives, malheureusement pas assez nombreuses, qui ont permis de définir des systèmes alimentaires dans de nombreux écosystèmes (par exemple Pagezy 1988, Robbe 1994, Koppert et al. 1996).

\section{Photo 1 : Enfants hmong déjeunant le matin}

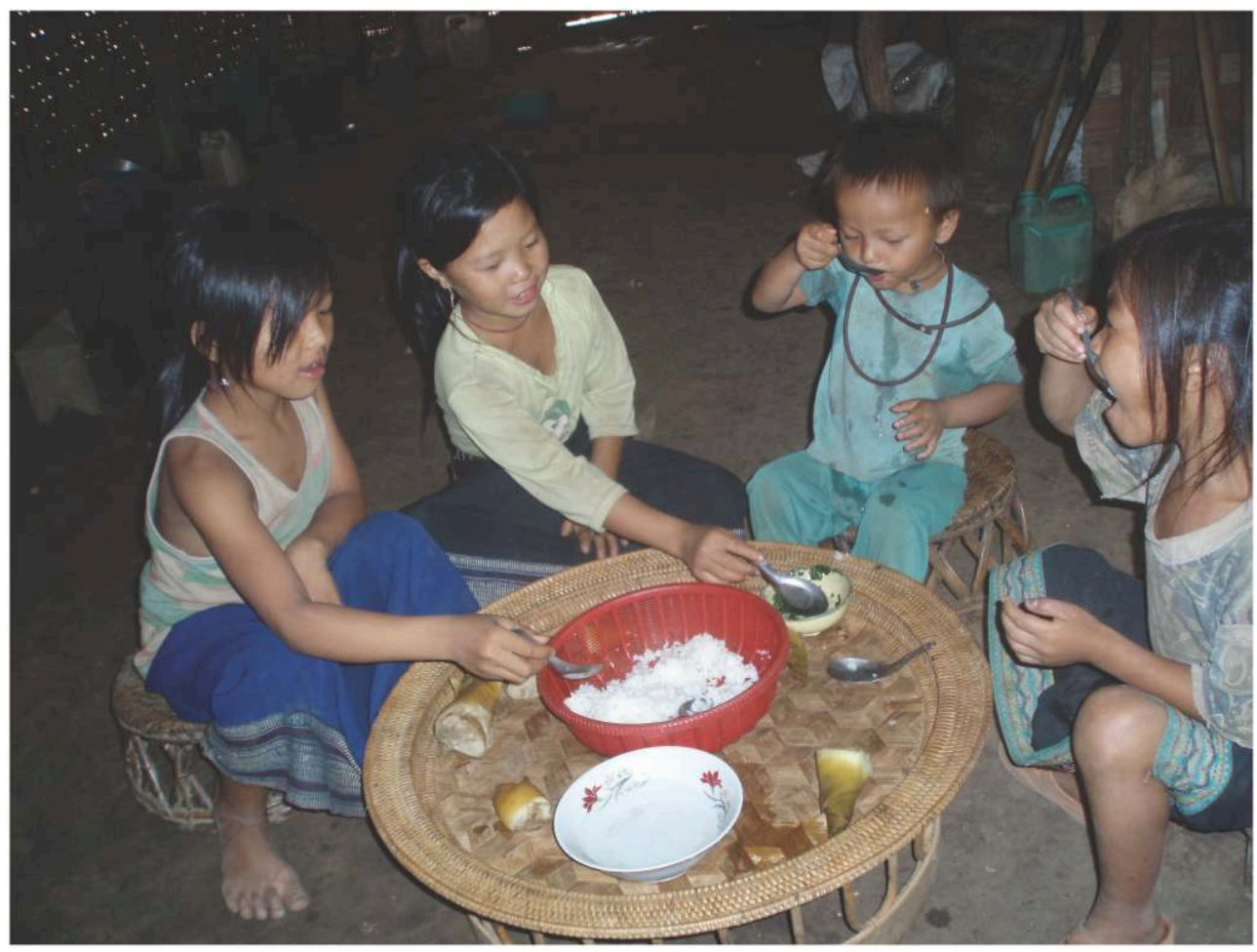

Le matin, pendant que les parents sont aux champs, les enfants hmong déjeunent de riz accompagné des pousses de bambous qu'ils ont ramassées la veille, trempées dans un peu dans le sel, et ils prennent l'eau fraîche à la petite cuillère. Laos, Pha Deng, 2011

Cliché F. Aubaile 
Photo 2 : Enfants pygmées

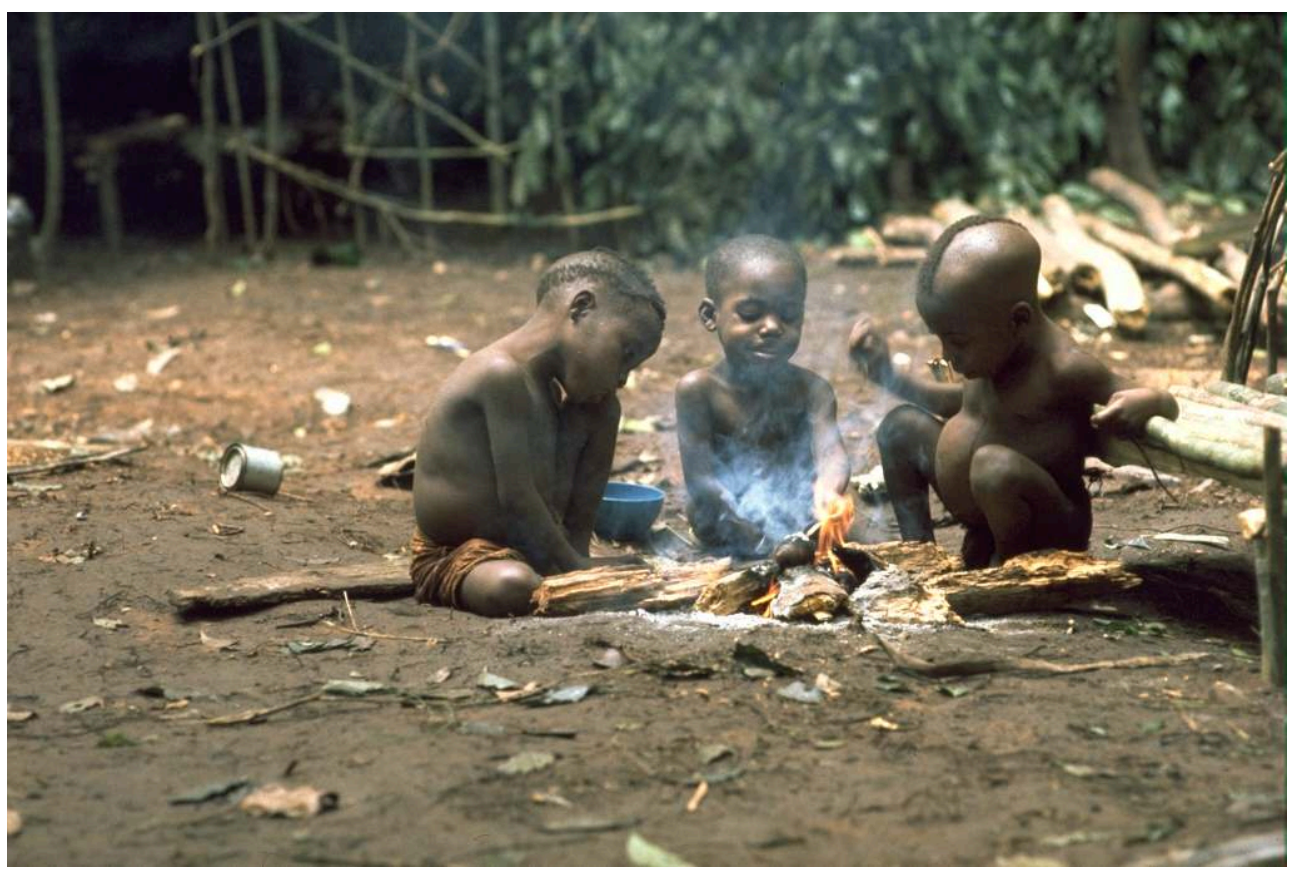

Dînette d'enfants aka avec un escargot achatine (chasseurs-cueilleurs de République centrafricaine)

Cliché S. Bahuchet

Actuellement, nous disposons donc des bases qui permettraient de tester, voire de mesurer, les changements. Il importe de réactualiser les données acquises précédemment, et de les étendre. Il faut aussi rappeler, comme cela a été fait tout au long du colloque, la nécessité absolue d'obtenir des données quantifiées et détaillées, au niveau du groupe comme des individus, et non pas se contenter de statistiques globales. C'est dans le détail que réside l'équilibre des régimes alimentaires, à travers l'apport de la biodiversité. Il convient aussi de documenter, tant qu'il en est encore temps, des modes alimentaires particuliers, tel que le régime carné des éleveurs semi-nomades de Kirghizie. C'est par exemple ce que s'est attaché à faire le programme de la FAO piloté par H. Kuhnlein sur les systèmes alimentaires des peuples indigènes (Kuhnlein et al. 2009). À l'évidence cette démarche doit être étendue. N'oublions pas, toutefois, combien le phénomène alimentaire ne peut être compris qu'en prêtant la plus grande attention, non seulement au détail du régime et de ses composants, mais aussi aux choix culturels et aux pratiques sociales qui le constituent (Garine 1990).

Un tel état des lieux, qui est à notre portée, permettra de mettre en évidence les pratiques sur le temps long cher à Braudel, tout en donnant des clefs de compréhension des lignes de transformation. Il nous apparaît que les critères identitaires sont à étudier finement, avec les phénomènes de mode et de distinction sociale, pour ce qu'ils entrent dans les forces et les tendances conduisant l'évolution - il est cependant possible que ces phénomènes offrent aussi des outils permettant de réinsérer la biodiversité dans les choix culturels en vue d'un régime alimentaire diversifié! Les brassages migratoires qui caractérisent le monde actuel peuvent offrir des «laboratoires » où observer et analyser le changement et surtout les processus d'emprunt et de transformation des pratiques alimentaires. 

monde!

\section{BIBLIOGRAPHIE}

Ahahan A. 2010 - Agriculture urbaine et stratégies de survie des ménages pauvres dans le complexe spatial du district d'Abidjan. Vertigo $2: 26 \mathrm{p}$.

Baudry S. 2011 - Les community gardens de New York City : de la désobéissance civile au développement durable. Revue Française d'Études Américanistes, 129 : 73-86.

Bellows A. C. \& Hamm M. W. 2001 - Local autonomy and sustainable development : Testing import substitution in more localized food systems. Agriculture and Human Values 18(3) : 271-284.

Bérard L. \& Marchenay P. 2004 - Les produits de terroir ; entre cultures et règlements. Paris, CNRS Éditions, 230 p.

Burlingame B., Charrondiere R. \& Mouille B. 2009 - Food composition is fundamental to the cross-cutting initiative on biodiversity for food and nutrition. Journal of food composition and analysis $22: 361-365$.

Castro Lara D., Basurto Peña F., Mera Ovando L. M. \& Bye Boettler R. A. 2011 - Los quelites, tradición milenaria en México. Universidad autonoma Chapingo, 37 p.

Dogan Y., Baslar S., Ay G. \& Mert H. H. 2004 - The use of wild edible plants in Western and Central Anatolia (Turkey). Economic Botany 58(4) : 684-690.

Flyman M. V. \& Afolayan A. J. 2006 - The suitability of wild vegetables for alleviating human dietary deficiencies. South African Journal of Botany 72 : 492-497.

Francis C., Lieblein G., Gliessman S., Breland T., Creamer N., Harwood R., Salomonsson L., Helenius J., Rickerl D. \& Salvador R. 2003 - Agroecology : the ecology of food systems. Journal of sustainable agriculture 22(3) : 99-118.

Frison E. A., Cherfas J. \& Hidgkin T. 2011 - Agricultural biodiversity is essential for a sustainable improvement in food and nutrition security. Sustainability $3: 238-253$.

Froment A., Garine I. de, Binam Bikoi C. \& Loung J.-F. (Ed.) 1996 - Bien manger et bien vivre. Anthropologie alimentaire et développement en Afrique intertropicale : du biologique au social. Paris, L'Harmattan, $520 \mathrm{p}$.

Galluzzi G., Van Duijvendijk C., Collette L., Azzu N. \& Hodgkin T. (Ed.) 2011 - Biodiversity for food and agriculture. Contributing to food security and sustainability in a changing world. Rome, FAO/ Platform for Agrobiodiversity Research, $66 \mathrm{p}$.

Garine I. de 1972 - The socio-cultural aspects of nutrition. Ecology of food and nutrition $1: 143-163$. Garine I. de 1990 - Les modes alimentaires : histoire de l'alimentation et des manières de tables. In Poirier J. (éd.), Histoire des mœurs I(2), Paris, Gallimard « La Pléiade » : 1447-1627. 
Garine E. 1996 - Une bonne sauce de mauvaises herbes : note sur les repas des Duupa du massif de Poli (Nord-Cameroun). In Bataille-Benguigui M.-C. \& Cousin F. (Ed.), Cuisines : Reflets des sociétés, Paris, Sépia/Musée de l'Homme : 75-96.

Golden C. D., Fernald L. C., Brashares J. S., Rasolofoniaina B. R. \& Kremen C. 2011 - Benefits of wildlife consumption to child nutrition in a biodiversity hotspot. Proceedings of the National Academy of Sciences 108(49) : 19653-19656.

Hladik C. M., Hladik A., Linares O. F., Pagezy H., Semple A. \& Hadley M. (Ed.) 1993 - Tropical forests, people and food: Biocultural interactions and applications to development. Paris, UNESCO-Parthenon, $876 \mathrm{p}$.

Johns T. \& Eyzaguirre P. B. 2006 - Linking biodiversity, diet and health in policy and practice. Proceedings of the nutrition society $65: 182-189$.

Johns T. \& Sthapit B. R. 2004 - Biocultural diversity in the sustainability of developing-country food systems. Food \& Nutrition Bulletin 25(2) : 143-155.

Kennedy G. \& Burlingame B. 2003 - Analysis of food composition data on rice from a plant genetic resources perspective. Food Chemistry $80: 589-596$.

Kennedy G., Nantel G. \& Shetty, P. 2003 - The scourge of "hidden hunger" : global dimensions of micronutrient deficiencies. Food Nutrition and Agriculture 32 : 8-16.

Koppert G., Rikong Adié H., Gwangwa'a S., Sajo Nana E., Matze M., Pasquet P., Froment A. \& Garine I. de 1996 - La consommation alimentaire dans différentes zones écologiques et économiques du Cameroun. In Froment A., Garine I. de, Binam Bikoi C. \& Loung J.-F. (Ed.) Bien manger et bien vivre. Anthropologie alimentaire et développement en Afrique intertropicale : du biologique au social. Paris, L'Harmattan : 237-254.

Kuhnlein H. V., Barthet V., Farren A., Falahi E., Leggee D., Receveur O. \& Berti P. 2006 - Vitamins $\mathrm{A}, \mathrm{D}$, and $\mathrm{E}$ in Canadian Arctic traditional food and adult diets. Journal of food composition and analysis 19(6) : 495-506.

Kuhnlein H. V., Erasmus B. \& Spigelski D. 2009 - Indigenous peoples' food systems : the many dimensions of culture, diversity and environment for nutrition and health. Rome, FAO, Centre for Indigenous Peoples' Nutrition and Environment, $\mathrm{x}+339$ p. + xxxviii p. of plates.

Muchnik J. 2012 - Sistemas agroalimentarios localizados : desarollo conceptual y diversidad de situaciones. In Torres Salcido G. \& Larroa Torres R. M. (Ed.) 2012 - Sistemas agroalimentarios localizados. Identidad territorial, construcción de capital social e instituciones. Mexico, UNAM/ Juan Pablos Editor : 25-42.

Norgan N. G., Ferro-Luzzi A., Durnin J. V. G. A. 1974 - The energy and nutrient intake and the energy expenditure of 204 New Guinean adults. Royal Society of London, PhiIosophical Transactions, série B, vol. 268 : 309-343.

Oomen H. A. P. C. 1971 - Ecology of human nutrition in New Guinea. Ecology of food and nutrition 1 : 1-18.

Pagezy H. 1988 - Coping with uncertainty in food supply among the Oto and the Twa living in the equatorial flooded forest near Lake Tumba, Zaïre. In Garine I. de \& Harrison G. A. (Ed.) Coping with uncertainty in food supply. Oxford, Oxford University Press : 175-209.

Petrini C. 2005 - Slow Food, manifeste pour le goût et la biodiversité. Barret-sur-Méouge, Éditions Yves Michel, 203 p. 
Picó B. \& Nuez F. 2000 - Minor crops of Mesoamerica in early sources (I). Leafy vegetables. Genetic Resources and Crop Evolution 47 : 527-540.

Ranfa A., Maurizi A., Romano B. \& Bodesmo M. 2013 - The importance of traditional uses and nutraceutical aspects of some edible wild plants in human nutrition : the case of Umbria (central Italy). Plant Biosystems [en ligne] DOI :10.1080/11263504.2013.770805, 10 p.

Richards A. I. 1932 - Hunger and work in a savage tribe, a functional study of nutrition among the southern Bantu. Londres, Routledge.

Robbe P. 1994 - Les Inuit d'Ammassalik, chasseurs de l'Arctique. Paris, Éditions du Muséum national d'Histoire naturelle, $390 \mathrm{p}$.

Rodahl K. 1964 - Les besoins nutritionnels dans la région polaire. Cahiers de santé publique 18 : 109-129.

Smith P. A. \& Smith R. M. 1999 - Diets in Transition : Hunter-gatherer to station diet and station diet to the self-select store diet. Human Ecology 27(1) : 115-133.

Tardıó J., Pascual H. \& Morales R. 2005 - Wild food plants traditionally used in the province of Madrid, Central Spain. Economic Botany 59(2) : 122-136.

\section{NOTES}

1. Tous les textes officiels sont disponibles sur le site de la Convention : http://www.cbd.int/ convention/text/.

2. Décision III/11. Les textes des décisions sont disponibles sur le site http://www.cbd.int/ decisions/.

3. Décision $\mathrm{V} / 5$

4. Décision VIII/23

5. http://www.slowfood.com/ (consulté le 19 mai 2013)

6. http://www.fao.org/news/story/fr/item/176160/icode/ (consulté le 19/5/2013)

7. http://www.incredible-edible-todmorden.co.uk/home (consulté le 19/5/2013)

8. http://icafood.eu/what_is_icaf.htm (consulté le 19 mai 2013) 